$N \sigma K-22-024-001$

A MATRIX METHOD FOR CALCULATING-THE SOURCE FUNCTION, MEAN INTENSITY, AND FLUX IN A MODEL ATMOSPHERE

Robert L. Kurucz

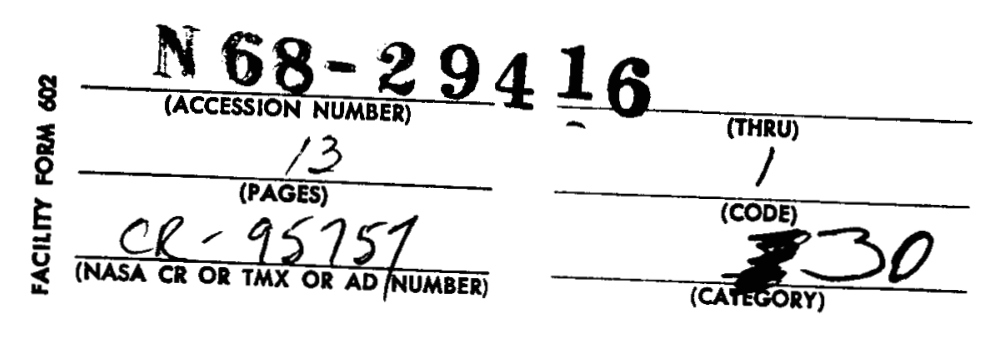

June 1968

GPO PRICE $\$$
CSFTI PRICE(S) $s$

Hard copy $(\mathrm{HC}) \frac{3.00}{.65}$

H 653 juiy 65

Smithsonian Institution Astrophysical Observatory

Cambridge, Massachusetts 02138 


\section{A MATRIX METHOD FOR CALCULATING THE SOURCE FUNCTION, MEAN INTENSITY, AND FLUX IN A MODEL ATMOSPHERE}

\section{ROBERT L. KURUCZ}

Smithsonian Astrophysical Observatory, Cambridge, Massachusetts and Harvard College Observatory, Cambridge, Massachusetts

\section{Received}

\section{ABSTRACT}

The integrals for the mean intensity and flux are expressed as the multiplication of the source-function vector by matrix representations of the integral operators. This method leads to a rapid solution of the integral equation for the source function and to an efficient calculation of the mean intensity and flux.

\section{INTRODUCTION}

In a model-atmosphere calculation the source function $S_{\nu}\left(\tau_{\nu}\right)$, the mean intensity $\mathrm{J}_{v}\left(\tau_{\nu}\right)$, and the flux $\mathrm{H}_{\nu}\left(\tau_{v}\right)$ must be evaluated for each iteration at each frequency $v$ and at each monochromatic optical depth ${ }_{\nu}{ }_{\nu}$ The mean intensity and flux are given by the integrals

$$
\begin{aligned}
& J_{v}\left(\tau_{\nu}\right)=\frac{1}{2} \int_{0}^{\tau} S_{v}(t) E_{1}\left(\tau_{v}-t\right) d t+\frac{1}{2} \int_{\tau_{v}}^{\infty} S_{v}(t) E_{1}\left(t-\tau_{\nu}\right) d t \text {, and } \\
& \mathrm{H}_{\nu}\left(\tau_{\nu}\right)=-\frac{1}{2} \int_{0}^{\tau_{\nu}} \mathrm{S}_{v}(\mathrm{t}) \mathrm{E}_{2}\left(\tau_{\nu}-\mathrm{t}\right) \mathrm{dt}+\frac{1}{2} \int_{T_{v}}^{\infty} \mathrm{S}_{v}(\mathrm{t}) \mathrm{E}_{2}\left(\mathrm{t}-\tau_{\nu}\right) d t,
\end{aligned}
$$


where $E_{n}(x)=\int_{1}^{\infty} e^{-x z / z^{n}} d z$ is the exponential integral of order $n$. The source function is given by the integral equation

$$
\mathrm{S}_{v}\left(\tau_{v}\right)=\left[1-a_{v}\left(\tau_{\nu}\right)\right] \mathrm{B}_{v}\left(\tau_{v}\right)+a_{\nu}\left(\tau_{v}\right) \mathrm{J}_{v}\left(\tau_{v}\right)
$$

where $a_{\nu}\left(\tau_{\nu}\right)$ is the fraction of opacity due to scattering and $\mathrm{B}_{v}\left(\tau_{\nu}\right)$ is the Planck function. The computations involved in the evaluation of $S_{v}$ and of the moment integrals $\mathrm{J}_{v}$ and $\mathrm{H}_{v}$ are extremely time consuming if the exponential integrals must be evaluated for every value of $\left|\tau_{\nu}-t\right|$. We will show below that these integrations can be expressed as the multiplication of the source-function vector by a matrix representation of the integral operator and that this approach leads to procedures that considerably reduce the integration time.

\section{DERIVATION OF THE INTEGRATION MATRIX}

The moment integrals of the specific intensity can be expressed (dropping the $v$ subscript) as

$$
M_{n}(\tau)=\frac{1}{2} \int_{0}^{\infty} \operatorname{sign}(t-\tau)^{n-1} S(t) E_{n}|t-\tau| d t
$$

Then,

$$
J(\tau)=M_{1}(\tau) \text { and } H(\tau)=M_{2}(\tau)
$$

We divide the integration range into $\mathrm{N}$ subintervals,

$$
M_{n \ell} \equiv M_{n}\left(\tau_{\ell}\right)=\frac{1}{2} \sum_{j=1}^{N} \operatorname{sign}\left(\tau_{j}-\tau_{\ell}\right)^{n-1} \int_{\tau_{j}}^{T+1} S(t) E_{n}\left|t-\tau_{\ell}\right| d t
$$


We now assume that $S$ can be represented by a parabola in the interval $\left(T_{j}, \tau_{j+1}\right)$. Thus,

$$
S(t)=\sum_{k=1}^{3} t^{k-1} \sum_{i=1}^{N} C_{j k i} s_{i}
$$

where the $C_{j k i}$ are interpolation coefficients. The $C_{j k i}$ can be, for example, the average of the forward-parabolic and the backward-parabolic interpolation coefficients. Inserting the expression for $S(t)$ into the jth term of the sum, we find that

$$
M_{n \ell j}=\frac{1}{2} \operatorname{sign}\left(\tau_{j}-\tau_{\ell}\right)^{n-1} \int_{\tau}^{\tau}{ }^{j+1} d t E_{n}\left|t-\tau_{\ell}\right| \sum_{k=1}^{3} t^{k-1} \sum_{i=1}^{N} C_{j k i} S_{i} .
$$

This expression can be simplified to

$$
M_{n \ell j}=\sum_{k=1}^{3} \eta_{n \ell j k} \sum_{i=1}^{N} C_{j k i} s_{i},
$$

where

$$
\eta_{n \ell j k}=\frac{1}{2} \operatorname{sign}\left(\tau_{j}-\tau_{\ell}\right)^{n-1} \int_{\tau_{j}}^{\tau_{j+1}} t^{k-1} E_{n}\left|\tau_{\ell}-t\right| d t
$$

is an integral that can be evaluated analytically. To evaluate $\eta_{n \ell j k}$ we use the indefinite integral

$$
\int E_{n}(x) d x=-E_{n+1}(x)
$$


and integrate by parts, obtaining

$$
\begin{aligned}
\eta_{n \ell j k} & =\frac{1}{2} \operatorname{sign}\left(\tau_{j}-\tau_{\ell}\right)^{n-1} \mid\left(\tau_{j}^{k-1} E_{n+1}\left|\tau_{\ell}-\tau_{j}\right|-\tau_{j+1}^{k-1} E_{n+1}\left|\tau_{\ell}-\tau_{j+1}\right|\right) \\
& +(k-1) \operatorname{sign}\left(\tau_{j}-\tau_{\ell}\right)\left(\tau_{j}^{k-2} E_{n+2}\left|\tau_{\ell}-\tau_{j}\right|-\tau_{j+1}^{k-2} E_{n+2}\left|\tau_{\ell}-\tau_{j+1}\right|\right) \\
& +(k-1)(k-2)\left(\tau_{j}^{k-3} E_{n+3}\left|\tau_{\ell}-\tau_{j}\right|-\tau_{j+1}^{k-3} E_{n+3}\left|\tau_{\ell}-\tau_{j+1}\right|\right) \mid
\end{aligned}
$$

We now collect terms to achieve the form of a matrix operator on $S$,

$$
\begin{aligned}
M_{n \ell} & =\sum_{j=1}^{N} \sum_{k=1}^{3} \eta_{n \ell j k} \sum_{i=1}^{N} C_{j k i} s_{i} \\
& =\sum_{j=1}^{N} \sum_{k=1}^{3} \sum_{i=1}^{N} \eta_{n \ell j k} C_{j k i} s_{i} \\
& =\sum_{j=1}^{N} \sum_{k=1}^{3} \sum_{i=1}^{N} \eta_{n \ell i k} C_{i k j} s_{j} \\
& =\sum_{j=1}^{N} \Xi_{n \ell j} s_{j},
\end{aligned}
$$

where

$$
\Xi_{n \ell j}=\sum_{k=1}^{3} \sum_{i=1}^{N} \eta_{n \ell i k} C_{i k j} .
$$

The matrix $\Xi_{\mathrm{n}}$ is the desired matrix operator

$$
M_{n}=\Xi_{n} S
$$

4 


\section{APPLICATIONS}

We define the matrices

$$
\Lambda \equiv \Xi_{1}
$$

and

$$
\Phi \equiv \Xi_{2}
$$

so that

$$
J=\Lambda S
$$

and

$$
\mathrm{H}=\Phi \mathrm{S} \text {. }
$$

The integral equation for $\mathrm{S}$ becomes

$$
S=(I-a) B+a \Lambda S
$$

or

$$
(I-a \Lambda) S=(I-a) B \text {, }
$$

where $I$ is the identity matrix and $a$ is now a diagonal matrix. The formal solution is

$$
S=(I-a \Lambda)^{-1}(I-a) B
$$

The solution of the integral equation is obtained by direct inversion, by Gauss-Jordan elimination, or by Gauss-Seidel iteration. The timings for these methods are approximately proportional, respectively, to $2 \mathrm{~N}^{3}, \mathrm{~N}^{3} / 2$, and the number of iterations times $\mathrm{N}^{2}$, where $\mathrm{N}$ is the dimension of the 
matrix. We have found the Gauss-Seidel method to be the fastest procedure for evaluating $S$ for the wide range of conditions encountered in modelatmosphere calculations.

The integration matrices $\Lambda$ and $\Phi$ can be pretabulated for some fixed $T$ set, where the values of $\tau$ are chosen to give accurate integrations. We can then evaluate $\mathrm{S}_{v}, \mathrm{~J}_{\nu}$, and $\mathrm{H}_{v}$ by interpolating $a_{\nu}$ and $\mathrm{B}_{v}$ from the $\tau_{\nu}$ set onto the fixed $T$ set, integrating, and then interpolating the resulting values back onto the $\tau_{\nu}$ set. Optical depths larger than the last value of the fixed $T$ set must be treated separately. However, for large depths this problem is straightforward, since the asymptotic behavior of $\mathrm{J}_{\nu}$ and $\mathrm{H}_{\nu}$ is particulary simple; namely,

$$
\mathrm{J}_{v}=\mathrm{S}_{v}+\frac{1}{3} \frac{\mathrm{d}^{2} \mathrm{~S}_{v}}{\mathrm{~d}^{2}} \quad \text { and } \quad \mathrm{H}_{v}=\frac{1}{3} \frac{\mathrm{d} \mathrm{S}_{v}}{\mathrm{~d} \tau_{v}}
$$

We illustrate this procedure in the following manner. Let the superscript in denote the internal fixed $\tau$ set and the superscript ex denote the external $\tau_{\nu}$ set. Furthermore, let $P$ be the interpolation operator from the $T_{\nu}$ set onto the fixed $T$ set and $Q$ be the operator from the fixed $\tau$ set back onto the $T_{\nu}$ set. The case where $S$ is prescribed and where we wish to find $\mathrm{J}$ and $\mathrm{H}$ can be expressed symbolically as follows:

First we interpolate in

$$
\mathrm{S}^{\text {in }}=\mathrm{PS}^{\mathrm{ex}} \text {; }
$$

then we integrate

$$
\begin{aligned}
& \mathrm{J}^{\text {in }}=\Lambda \mathrm{S}^{\text {in }} \\
& \mathrm{H}^{\text {in }}=\Phi S^{\text {in }} ;
\end{aligned}
$$


and finally we interpolate out

$$
\begin{aligned}
& J^{e x}=Q J^{i n} \\
& H^{e x}=Q H^{i n}
\end{aligned}
$$

or

$$
\begin{aligned}
& J^{e x}=Q \Lambda P S^{e x} \\
& H^{e x}=Q \Phi P S^{e x} .
\end{aligned}
$$

The case where $a$ and $B$ are prescribed and where we wish to find $S, J$, and $\mathrm{H}$ can be symbolized as follows:

We interpolate in

$$
\begin{aligned}
& a^{i n}=P a^{e x} \\
& B^{i n}=P B^{e x},
\end{aligned}
$$

integrate

$$
\begin{aligned}
& S^{\text {in }}=\left(I-a^{i n} \Lambda\right)^{-1}\left(I-a^{i n}\right) B^{i n} \\
& J^{i n}=\Lambda S^{i n} \\
& H^{i n}=\Phi S^{i n},
\end{aligned}
$$

and interpolate out

$$
\begin{aligned}
& S^{e X}=Q S^{i n} \\
& J^{e x}=Q J^{i n} \\
& H^{e x}=Q H^{i n} .
\end{aligned}
$$


If the interpolation operators are matrices, then $Q \Lambda P$ and $Q \Phi P$ can be considered the integration operators $\Lambda^{\mathrm{ex}}$ and $\Phi^{\mathrm{ex}}$ on the $\tau^{\mathrm{ex}}$ set, so that

$$
\mathrm{J}^{\mathrm{ex}}=\Lambda^{\mathrm{ex}} \mathrm{S}^{\mathrm{ex}}
$$

and

$$
H^{e x}=\Phi^{e x} S^{e x}
$$

However, the calculation of these external operators is slower than if we simply interpolate in and out, because two additional matrix multiplications are needed.

The author's model-atmosphere program ATLAS uses the matrix methods described above to calculate $\mathrm{S}_{v}, \mathrm{~J}_{v}$, and $\mathrm{H}_{v}$. These calculations are made with 40 values of $\tau^{e x}$ and 40 values of $\tau^{\text {in }}$. The interpolation routine uses a weighted average of forward-and backward-parabolic interpolation coefficients. The integral equation for the source function is solved by use of a modified Gauss-Seidel iteration that is terminated when the maximum relative change is less than $10^{-5}$. This accuracy is sufficient for $\mathrm{S}^{\text {in }}$, since the accuracy of the parabolic interpolation is somewhat less than this when only 40 points are used.

Figures 1 and 2 show the convergence properties of this method for two calculations of $\mathrm{S}_{v}$ in a model atmosphere with effective temperature $10000^{\circ} \mathrm{K}$ and $\log g$ of 2 . Figures 3 and 4 show the corresponding values of $1-a_{v}$, $\mathrm{B}_{v}, \mathrm{~S}_{v}, \mathrm{~J}_{v}$, and $\mathrm{H}_{v}$. This model provides a good test of the Gauss-Seidel method because scattering is a large fraction of the opacity at this low gravity, where hydrogen is mostly ionized. Only at frequency points where scattering exceeded 99.99 per cent at the surface were more than four Gauss-Seidel iterations required. 
The author wishes to thank Dr. Wolfgang Kalkofen for suggesting this investigation. Mr. Deane Peterson and Dr. Stephen Strom contributed helpful criticism and many useful discussions to this work. Support for the computations reported in this work was provided, in part, by grant NGR 22-024-001 from the National Aeronautics and Space Administration. The author also acknowledges support from the SAO fellowship program. 


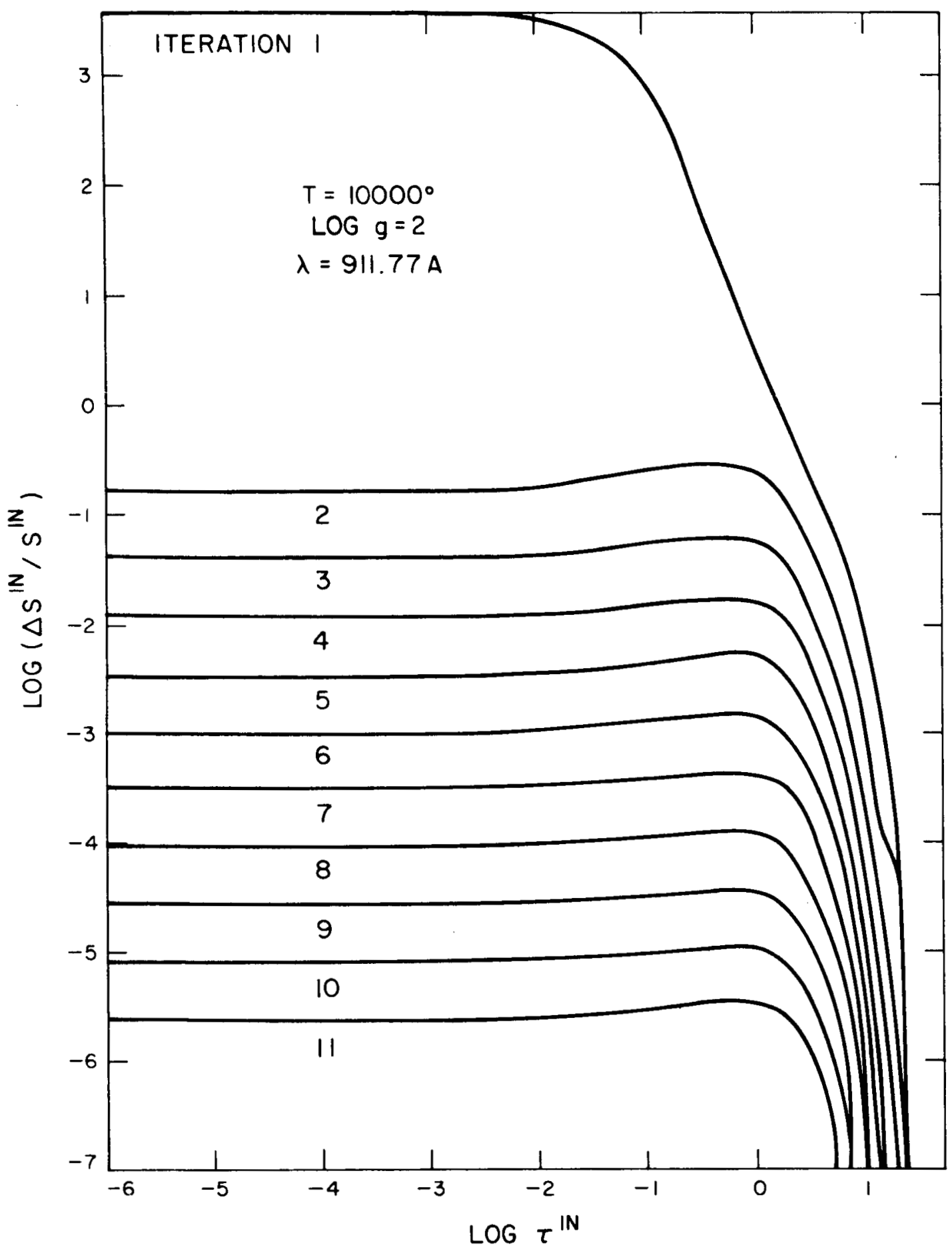

Fig. 1. - Relative change for each subiteration of the source-function calculation at the long-wavelength side of the Lyman discontinuity. Here the scattering fraction is 0.9999 near the surface and $>0.6$ at large depths. 


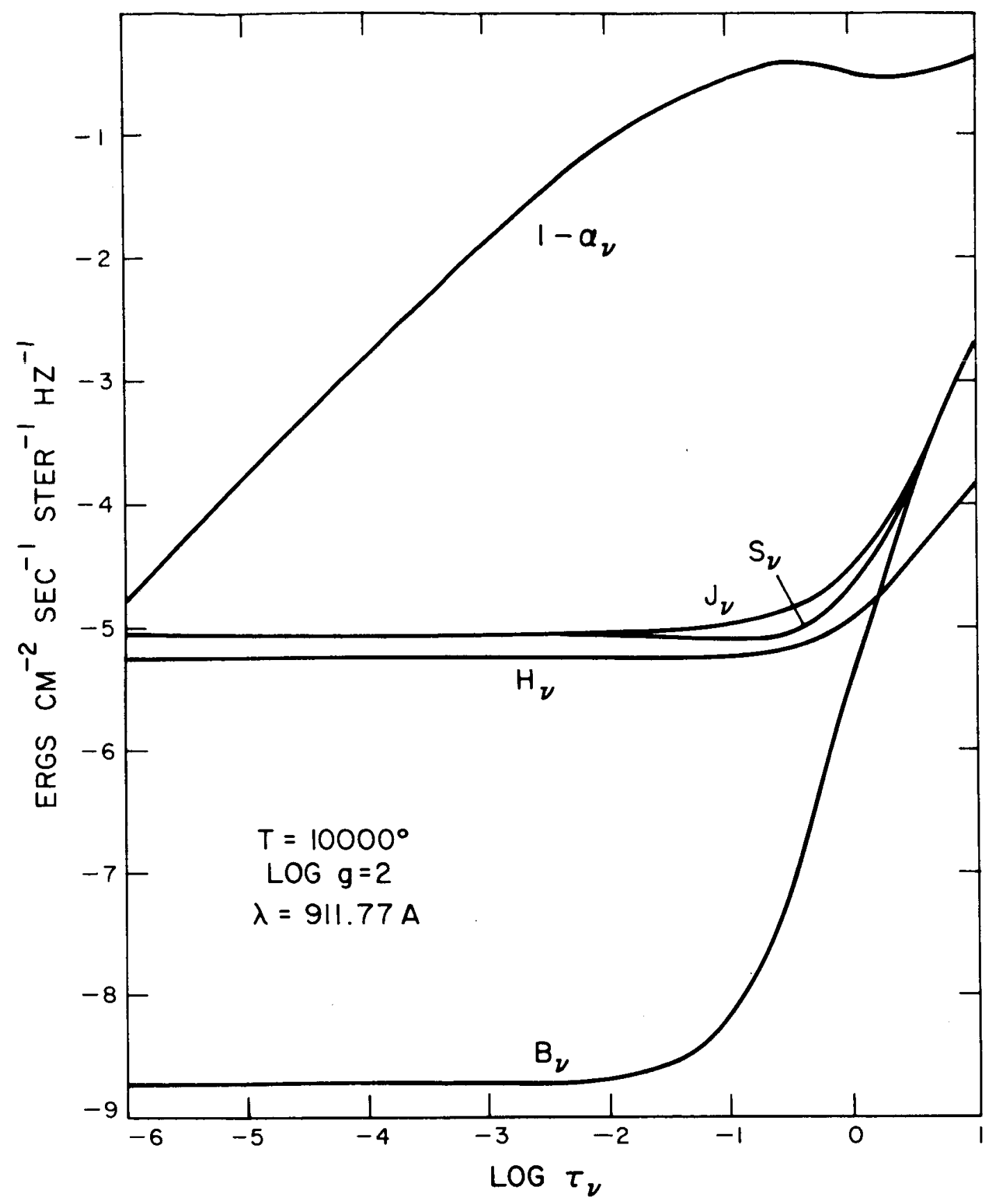

Fig. 2. - The input scattering fraction, Planck function, and the resulting source function, mean intensity, and flux corresponding to the source-function calculation shown in Figure 1. 


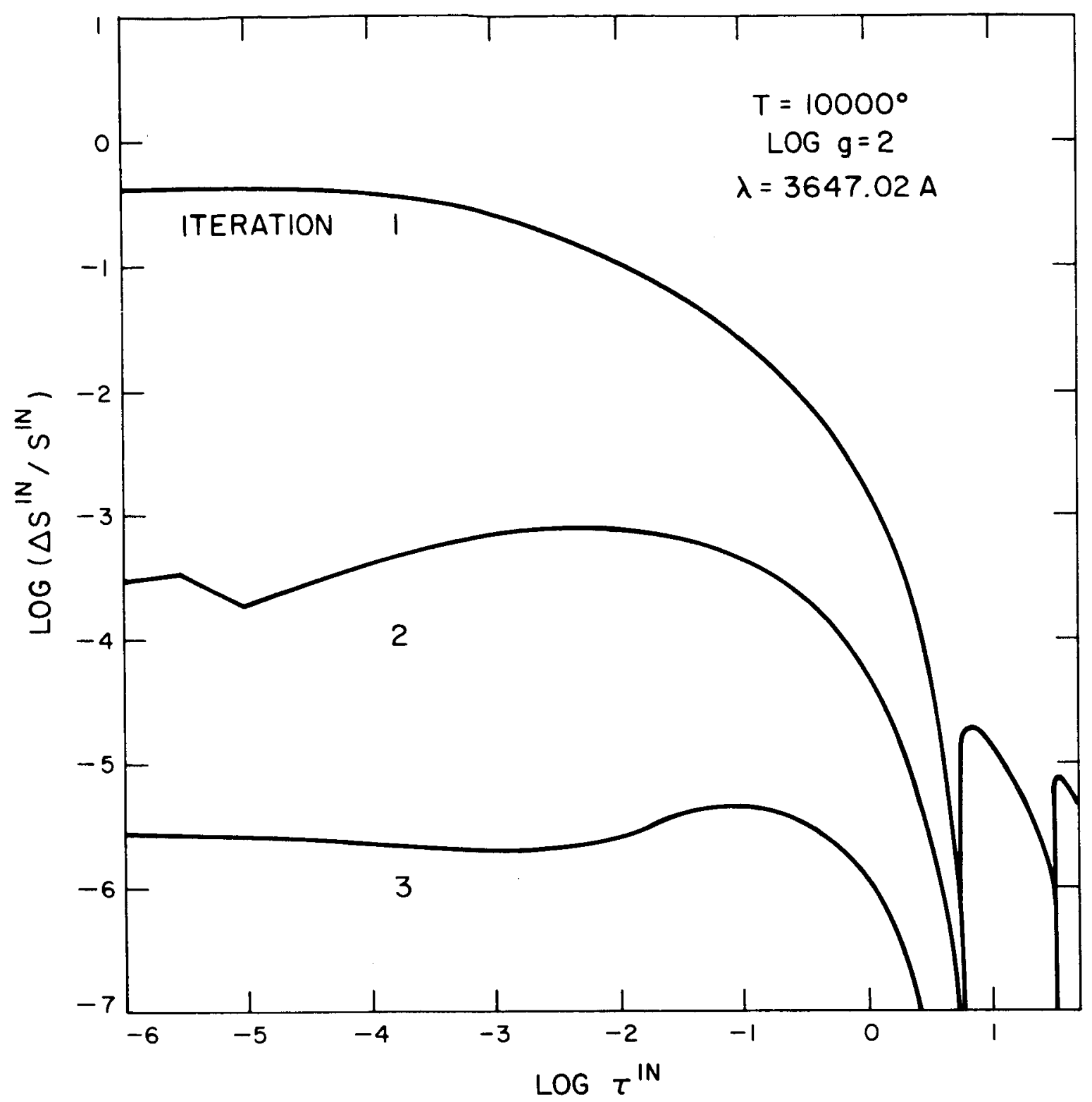

Fig. 3. - Same as Figure 1, for the short-wavelength side of the Balmer discontinuity where there is considerably less scattering, i.e., 0.03 at large depths. 


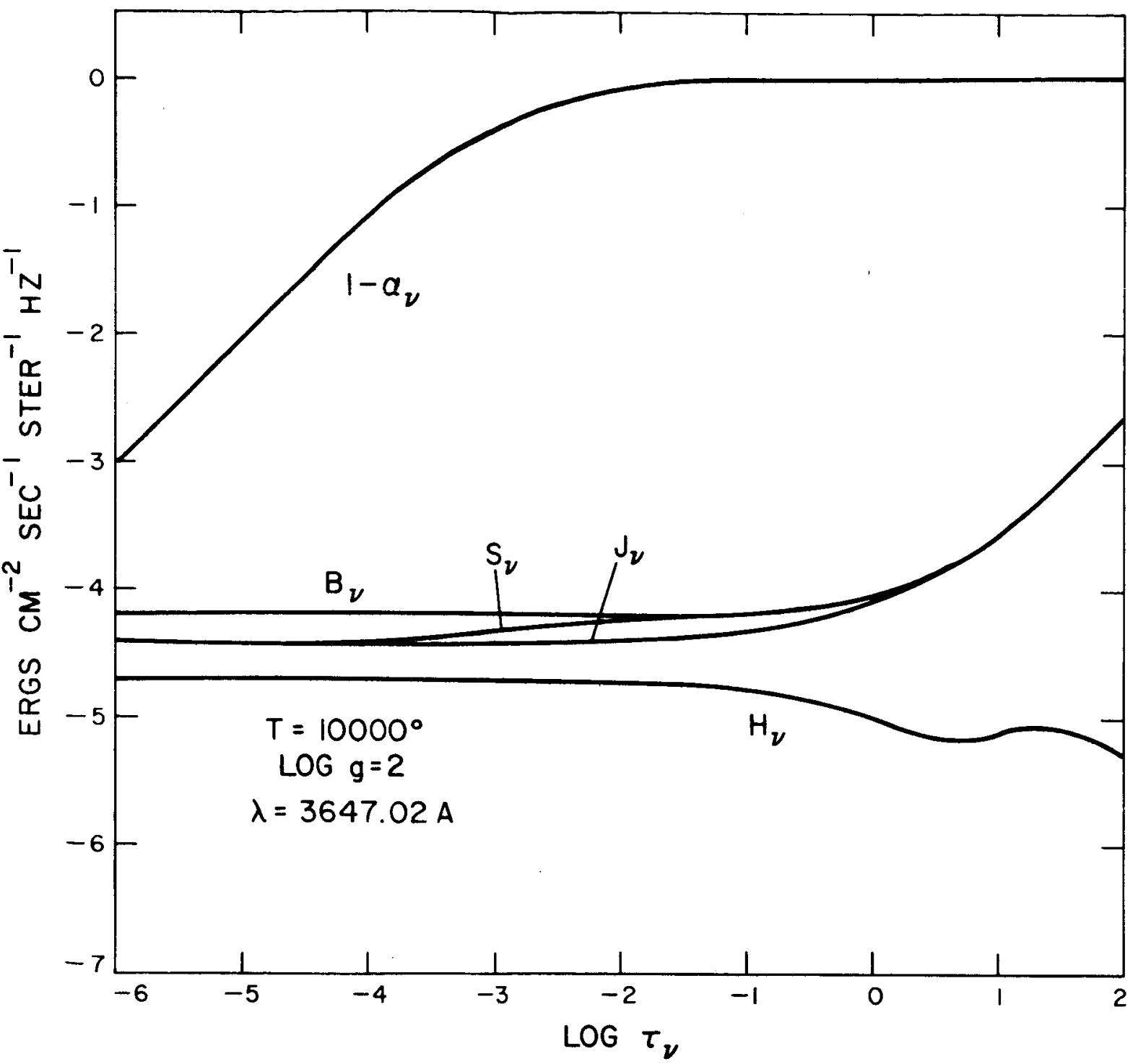

Fig. 4. - Same as Figure 2, for the short-wavelength side of the Balmer discontinuity where there is considerably less scattering, i. e., 0.03 at large depths. 